\title{
Determination of butyric acid dosage based on clinical and experimental studies - a literature review
}

\author{
Tomasz Banasiewicz', Dorota Domagalska ${ }^{1}$, Katarzyna Borycka-Kiciak², Grażyna Rydzewska ${ }^{3,4}$ \\ ${ }^{1}$ Chair and Clinic of General and Endocrine Surgery and of Gastrointestinal Oncology, Poznan University of Medical Sciences, \\ Poznan, Poland \\ ${ }^{2}$ Clinic of General and Colorectal Surgery, Centre of Postgraduate Medical Education, Bielanski Hospital, Warsaw, Poland \\ ${ }^{3}$ Clinic of Internal Diseases and Gastroenterology, Central Teaching Hospital of the Ministry of Interior and Administration \\ (MSWiA), Warsaw, Poland \\ ${ }^{4}$ Faculty of Medicine and Health Sciences, Jan Kochanowski University, Kielce, Poland
}

Gastroenterology Rev 2020; 15 (2): 119-125

DOI: https://doi.org/10.5114/pg.2020.95556

Key words: butyrate, irritable bowel syndrome, dosage, short-chain fatty acids.

\begin{abstract}
Address for correspondence: Dorota Domagalska MD, Chair and Clinic of General and Endocrine Surgery and of Gastrointestinal Oncology, Poznan University of Medical Sciences, 49 Przybyszewskiego St, 60-355 Poznan, Poland, phone: +48 7819396 46,
\end{abstract} e-mail: dorotablazejewska@gmail.com

\begin{abstract}
Short-chain fatty acids produced by bacteria living in the large intestine are the main energy substrate for the colonocytes. Butyric acid is used for the treatment and prevention of exacerbations of various gastrointestinal diseases: diarrhoea, intestinal inflammations, functional disorders, dysbiosis, and post-surgery or post-chemotherapy conditions. The current standard doses of butyric acid (150-300 $\mathrm{mg}$ ) range between $1.5-3 \%$ and $15-30 \%$ of the reported daily demand. Increased metabolism of the colonocytes in conditions involving intestine damage or inflammation, increased energy expenditure during a disease, stimulation of intestine growth in 'stress' conditions with accelerated intestinal passage and increased intestinal excretion, and decreased production of endogenous butyrate due to changes in bacterial flora in different pathological conditions require a significant increase of the supply of this acid. Physiological high demand for butyrate and known mechanisms of pathological conditions indicate that current supplementation doses do not cover the demand and their increase should be considered.
\end{abstract}

Short-chain fatty acids (SCFA) are produced by bacteria dwelling in the large intestine. They are a product of the metabolism of polysaccharides that are not digested by the digestive system enzymes. At the same time, they are the main energy substrate for the epithelial cells of the intestinal mucosa. More and more scientific reports focus on the significance of SCFA and in particular that of butyric acid [1]

Butyric acid present in the lumen of the gastrointestinal tract is indispensable for maintenance of normal homoeostasis of the mucosa cells. It conditions their normal metabolism (as the basic source of energy) and proliferation, and it is responsible for regeneration and repair processes. It stimulates local cellular response, maintains intestinal barrier integrity, and inhibits tumour cell differentiation [2, 3]. It also has a favourable effect on the intestinal microbiome [1] by stimulation of the growth of the saprophytic flora and by an inhibitory effect on the development of other pathogens, such as Escherichia coli, Campylobacter, or Salmonella [4].

Butyric acid is increasingly used as a supportive agent in the treatment and prevention of exacerbations of various diseases and disorders of the digestive tract, such as diarrhoea (specific and non-specific), inflammatory conditions (non-specific bowel inflammation, diverticulitis, diversion colitis, radiation-induced bowel inflammation), functional disturbances (irritable bowel syndrome), dysbiosis, and post-surgery (resections, short bowel syndrome) or post-chemotherapy conditions. Recently, it has been stressed that SCFA affect not only processes occurring in the lumen of the gastrointestinal tract but also other systems and organs, such as circulatory or nervous systems, through mechanisms associated with the intestinal barrier, carbohydrate metabolism, immunomodulation, and appetite control, and with an effect on obesity [5]. 
150-300 mg/day is the most common dosage recommendation for currently available butyric acid products. It is not easy to determine the optimal dose of butyric acid supplementation, and the results of the studies conducted to date are often highly inconclusive. High viscosity of the intestinal contents, the presence of bacterial biofilm and mucus layer on the mucosa surface, and rapid absorption of SCFA make it difficult to determine their concentration on the mucous membrane surface itself. On the other hand, SCFA concentration in the intestinal lumen or faeces does not reflect the rate of their production [6].

Physiologically, butyric acid, like other SCFA, is a product of anaerobic bacterial fermentation of resistant starch and food fibres. The total concentration of SCFA in the intestinal lumen varies between 60 and $150 \mathrm{mmol} / \mathrm{kg}$, and their daily production in the large intestine of a healthy individual is $300-400 \mathrm{mmol}[4,7]$. This translates into $50-70 \mathrm{mmol}$ of butyric acid or about 5.5-7.5 g/day. According to some studies, the physiological range of butyrate concentrations in the intestinal lumen is between 1 and $10 \mathrm{mmol} / \mathrm{l}$ of the food content [8], which, assuming a daily production of 9 । of the intestinal content, corresponds to $9-90 \mathrm{mmol} /$ day, i.e. 1-10 g/day. The daily demand for butyric acid in physiological conditions falls therefore within a very wide range of $1000 \mathrm{mg} /$ day to as much as $10,000 \mathrm{mg} /$ day, which should be covered by fermentation processes of resistant starch and food fibres. However, in the Western population insufficient supply of these nutrients is observed, which can explain the rapidly growing incidence of all types of gastrointestinal diseases, both inflammatory, neoplastic, and functional. In studies with significantly increased supply of fibre in the diet growth of probiotic bacteria such as Bifidobacterium (BfB) and Lactobacillus ( $L a b)$, improved condition of the intestinal mucosa, and even reduced risk of cancer of the lower colon segments were observed [9]. It seems that similar effects may be obtained by increased supplementation with butyric acid products.

In the analysis of the daily demand for butyric acid it is noticeable that the currently used standard doses of $150-300 \mathrm{mg}$ represent only $15-30 \%$ of the lowest daily demand reported, or they are even as low as 1.5-3\% if the highest possible values are taken into consideration. Thus, even under physiological conditions the current supplementation doses are low or even very low, and their increase may be considered. The possibility of dose increase depends on the formulation of butyric acid because packing more than $300 \mathrm{mg}$ of butyrate into a single capsule is a technical limitation here. Of course, the dose of "clean" (non-enveloped) butyrate may be increased, but this form is absorbed already in the upper gastrointestinal tract and reaches the intestine at a much lower concentration. Therefore, to achieve effective supplementation (with adequately high butyrate doses), the forms of butyrate supply should be optimised.

Animal studies showing beneficial trophic or anti-inflammatory effects are usually carried out with use of much higher doses of butyric acid. In an experiment where mice were administered $11 \mathrm{~g}$ of butyrate in drinking water per day (i.e. about $55 \mathrm{~g} / \mathrm{kg}$ of body weight!) an improvement of the immune functions of the intestinal epithelium was shown, which, according to the authors, may be an important mechanism of prevention of several chronic diseases [10]. In another study, conducted on birds, butyrate was used at a dose of $1000 \mathrm{mg} / \mathrm{kg}$ of body weight, and a reduction of interleukin 6 (IL-6) and tumour necrosis factor $\alpha$ (TNF- $\alpha$ ) levels as well as increased activity of peroxide dismutase and of catalase were observed [11]. This confirms an anti-inflammatory effect of butyrate. Of course, animal test results cannot be directly translated into human test results; however, much higher doses of butyrate are used also in clinical trials. An example is the above-mentioned publication with a dose of $4 \mathrm{~g}$ of butyrate per day in obese and healthy patients [12]. Efficacy of high doses was confirmed by a study in which healthy individuals received enemas containing $100 \mathrm{mmol}$ of butyrate. Anti-inflammatory effect, an increase in glutathione antioxidation, and a decrease of uric acid production were achieved in this study [13]. High doses of butyrate may thus have a favourable metabolic effect as well because they may exert epigenetic action [14]. Very good clinical results of high doses of butyrate $(2000 \mathrm{mg} /$ day) were found in patients with mild to moderate Crohn's disease [15]. A very good clinical effect was found in the majority of patients, as well as remission of lesions on endoscopic examination and a decrease of white blood cells (WBC) counts and nuclear factor $\kappa \mathrm{B}(\mathrm{NF}-\kappa \mathrm{B})$ and IL-1 $\beta$ activity.

However, particular attention should be paid to conditions with an increased demand for SCFA. They will be described below, but their common features include an increased demand for butyric acid on one hand, and reduction of its endogenous production on the other (lack of appetite, dietary restrictions, limited volume of the eaten food, malabsorption, disturbances in the composition of the saprophytic intestinal flora responsible for physiological fermentation processes). Taking into consideration the increased demand with concurrent reduction of endogenous production, additional supplementation with butyric acid seems particularly important for the achievement of the expected optimal clinical outcomes. 
Causes of increased demand:

1. Enhanced metabolism of intestinal epithelial cells in conditions with damage/inflammation of these cells.

2. Increased energy expenditure of the organism in pathological conditions.

3. Stimulation of intestine growth in 'stress' situations

4. Conditions with accelerated intestinal passage and increased secretion into the lumen of the intestine (diarrhoea).

5. Decreased production of endogenous butyric acid due to changes in bacterial flora in different pathological conditions.

6. Other conditions associated with increased demand for butyric acid in the digestive tract.

1. Butyric acid is used by intestinal epithelial cells, especially in processes associated with their intensive proliferation, related to inflammation, damage, and subsequent repair processes. It is difficult to quantify the level of this increase in demand, and there are few data in the subject literature. However, some analogies may be assumed, where in tissue and wound regeneration, metabolic demand increases by a factor of 1.6-2.0 with respect to the basic demand. It should be borne in mind that the energy demand of the intestinal epithelial cells is mainly covered by energy substrates available in the intestinal lumen, i.e. predominantly by butyric acid. The dose of butyric acid should therefore be increased accordingly during inflammatory conditions. This finds confirmation in clinical studies. In one of the studies on the treatment of ulcerative colitis butyric acid was used in the form of enema at doses of $40 \mathrm{mmol} / \mathrm{l}$ to up to $100 \mathrm{mmol} / \mathrm{l}$, which corresponds to $4.4-11 \mathrm{~g} / \mathrm{l}$. For $200 \mathrm{ml}$ enemas this translates into $800-2200 \mathrm{mg} /$ enema. Very good clinical outcomes were observed with these doses, with no side effects [16]. Similar observations were made in patients with a related condition, who received sodium butyrate enemas at a dose of $8800 \mathrm{mg} / \mathrm{l}$ with very good effect [17]. In animal studies, significantly higher doses of butyrate are used successfully. In one of these studies, supplementation with butyrate was used in pigs with induced ulcerative colitis. With use of butyrate at a dose of $1000 \mathrm{mg} / \mathrm{kg}$ body weight, a significant preventive effect with respect to intestine damage was achieved, through inhibition of apoptosis, improvement of tight junctions between cells, which improved the integrity of the intestinal barrier and activation of the endothelial growth factor (EGF) that stimulates regeneration processes [18]. This confirms the hypothesis that inflammation and regeneration conditions in the gastrointestinal tract causing a sig- nificant increase of energy demand of mucosal cells [19] may require higher doses of butyrate.

2. In most diseases and pathological conditions, especially those with regeneration, healing, and proliferation processes, the demand of the entire organism is increasing. If a patient undergoes surgical treatment, this should be taken into account in the calculations, and the demand for energy should be multiplied by a factor of 1.2 for patients after medium-extent surgical procedures (laparoscopy), by a factor 1.6 for patients after more extensive procedures, and even by a factor of 1.8 in case of large wounds with exudate, inflammatory reactions, or infections [20]. The energy demand of different organs varies. The intraperitoneal organs, including intestines, pancreas, spleen, and stomach, which account for $6 \%$ of the body's weight, use $20-35 \%$ of the body's total energy demand, and the intestines themselves - about 12-20\% [21]. In overweight patients, in patients with concurrent diseases, and in cachectic patients, energy demand of the visceral organs may increase at various treatment stages and may be difficult to cover [22]. A small proportion of this demand comes from blood vessels, and the vast majority of this demand is covered by SCFA. This indicates, on one hand, the importance and role of enteric nutrition, and on the other hand it justifies an increase in the dose of butyrate for all indications in patients with increased energy demand (patients after trauma or surgery, patients on rehabilitation or practising intensive exercise, cachectic patients, and cancer patients).

3. In the recent years, a very interesting observation emerged, that during various pathological and energy-consuming processes (surgical procedures, exposure to low temperatures, lactation, restrictions in caloric supply) the cells of the mucous membrane are stimulated to grow and proliferate. This may be a compensatory mechanism whose aim is to improve absorption and to prepare the body for better, i.e. more efficient, use of the food provided. The mechanism of this phenomenon is complex and still under investigation. One of the explanations is increased metabolism of the white fatty tissue, which induces secretion of a number of mediators (probably including leptin) that stimulate, via hypothalamus, intestine growth and cause increased supply of food (through the sensation of hunger) [23]. Experimental models have also shown that deficits in energy supply ('caloric restriction') lead to intestine growth, both quantitative (increase in organ mass and size) and qualitative (increase in intestinal cell amount) [24]. A very interesting study was conducted in a group of overweight patients with metabolic syndrome. Within 
that study, both the study group and a healthy control group received $4000 \mathrm{mg}$ of sodium butyrate per day. Decreased inflammatory activity of several investigated molecules and monocytes was observed in that study, which was particularly evident in the group of obese patients. This high dose of butyrate (almost eight times higher than the maximum dose currently recommended for butyric acid products available on the Polish market) showed a positive anti-inflammatory and immunomodulatory effect without side effects or adverse reactions [12].

In various pathological conditions, the demand of intestinal epithelial cells for energy can therefore increase significantly, due to intense proliferation of intestinal epithelium, i.e. increasing number of cells requiring energy substrates (butyric acid among them, to a large extent), high metabolism level, and concurrent increase in energy demand of the other organs. Because of the above, intestinal epithelial cells must cover the highest possible proportion of the increased energy demand by using substrates available in the digestive tract, i.e. mainly butyric acid, which justifies an increase in its supply.

4. In many situations, intestinal passage is accelerated and there is increased secretion into the intestinal lumen. This is associated, on one hand, with disturbances of the absorption ability of the intestinal epithelial cells, and on the other hand - with their increased energy expenditure. Another important consequence is decreased production of endogenous SCFA, because there is not enough time for natural fermentation of resistant starch and food fibre to occur. A significant reduction of SCFA production in patients with antibiotic-induced diarrhoea may be an example here. The use of antibiotics itself, without concurrent diarrhoea, also caused a decrease of SCFA levels, including the level of butyric acid, when dicloxacillin, erythromycin, and combined intravenous therapy with ampicillin and metronidazole were used [25]. The use of antibiotics, through their negative effect on the saprophytic intestinal flora, is a factor that significantly impairs the ability of intestinal epithelial cells to cover their energy demand in a physiological manner. This effect is greater during diarrhoea, which accelerates the passage time. This is confirmed by studies showing a favourable effect of supplementation with selected fatty acids in patients with travellers' diarrhoea, including butyric acid at a dose of $1350 \mathrm{mg} /$ day in the form of sodium butyrate [26]. A very good clinical effect was found in that study, without side effects or adverse reactions.

The clinical situations described above may therefore be an indication and justification for an increase of the dose of butyric acid supplementation in this group of patients.

5. A normal level of endogenous butyric acid production, as well as of other SCFA, depends on the physiological intestinal flora. Various types of microbiome disturbance may lead to a significant decrease of SCFA production [25]. Adverse changes within the intestinal microbiome may occur in several other conditions, often apparently unrelated. In a model of induced stroke in monkeys, significant changes in the intestinal microbiome were found; first of all, decreased amounts of Faecalibacterium, Oscillospira and Lactobacillus. This resulted in a significant decrease of endogenous fatty acid levels [26, 27]. This study shows, on one hand, how apparently unrelated conditions can negatively affect the SCFA level, and on the other hand it confirms the complexity of the intestine-brain axis mechanism. It is difficult to draw far-reaching clinical conclusions, but these are the mechanisms that can explain very frequent diagnosis of functional disorders of the gastrointestinal tract in neurological patients. Recent studies demonstrating that in patients with Alzheimer's disease a significant decline in fermenting bacteria and associated endogenous SCFA levels occur [28] indicate, on one hand, how complex the mechanisms leading to the SCFA deficit may be, and, on the other hand, how important SCFA supplementation in neurological diseases can be. The decrease of endogenous SCFA levels may justify an increase of the dose of butyrate supplementation.

6. The above-mentioned conditions associated with increased energy demand decreased the production of endogenous SCFA or their increased loss do not, of course, cover the entire broad spectrum of clinical situations where the demand for butyric acid rises. First of all, it should be recognised that a significant proportion of SCFA, including butyric acid, is absorbed along the entire length of the digestive tract. Of course, commercially available products protect butyrate in special matrices, but always some part of it will be absorbed earlier, and most of the diseases requiring butyric acid supplementation affect the lower digestive tract, including its distal parts. Studies with administration of butyric acid to chickens showed its very high absorption already at the level of the duodenum. A favourable trophic effect, growth stimulation, and optimisation of the function of the duodenal mucosa were found. Therefore, this seems to justify an increase in butyrate supplementation dose, as, on one hand, pathological conditions may also increase absorption in the upper part of the digestive tract and, on the other hand, provision of adequately high 
doses to the lower gastrointestinal tract should be aimed at [29]. Publications showing a role of SCFA after surgeries of the gastrointestinal tract (resections, anastomoses) as well as of the abdominal cavity (laparotomy, peritonitis, pancreatitis) are very interesting. The decrease in SCFA (levels), including butyric acid (levels), has been shown to have a negative effect on the integrity of the intestinal barrier and the tightness (normal healing) of anastomoses [30]. The conditions that are indication for surgery (inflammatory conditions, cancers) increase the demand for butyric acid, and surgery additionally raises this demand in a specific (anastomosis healing) and non-specific (increased energy demand due to surgical trauma and healing) way. Taking the above into consideration, it should be assumed that supplementation with high doses of butyrate in this group of patients is fully justified, both in the preoperative and in the postoperative period.

Supplementation with increased butyric acid doses seems to be particularly important in patients using substances, especially in cigarette smokers and alcohol drinkers, as well as in patients with other metabolic diseases, such as diabetes.

Alcohol significantly disturbs the intestinal microbiota, the final result of which is a significant decrease in production of endogenous fatty acids. Alcohol is also a factor that increases oxidative stress and increases the production of a number of its associated cytokines, such as tetradecane [31]. Decreased production of SCFA is also noted in patients smoking cigarettes, in whom microbiome changes are observed, predominantly in the form of decreased counts of fermenting bacteria responsible for the production of endogenous SCFA [32]. Smoking is also a factor that directly damages the mucous membrane of the gastrointestinal tract [33], which activates energy-consuming regeneration and repair processes and justifies supplementation with increased doses of butyrate. Particular attention should be paid to the appropriate dose of butyrate in persons who are both alcohol consumers and smokers [33].

Metabolic diseases also affect the level of endogenous fatty acids, which may lead both to a decrease in their content in the lumen of the digestive tract and to an increase in demand. Diabetes may be one of the examples, where significant microbiome changes in microbiome and decreased production of butyric acid are observed [34]. On the other hand, it is known that the supply of endogenous butyric acid can have a positive effect on the normalisation of bacterial flora [35], as well as on the efficiency of the immune system [36].

In light of available literature and pharmacological and clinical data, it should be concluded that butyric acid is a safe drug, with a very high safe and tolerated dose. In clinical conditions, it is practically impossible to overdose butyrate in a patient, both during drug studies and when used by a patient. No clinical side effects were observed in healthy volunteers administered therapeutic doses. No toxicity or adverse effects were observed in patients in clinical trials with a mixture of SCFA in the form of enemas containing from $40 \mathrm{mmol} / \mathrm{l}$ to $100 \mathrm{mmol} / \mathrm{l}[16,17]$. In clinical trials with oral drug administration, the safety of the use of butyrate and the absence of side effects, as well as its fully physiological mechanism of action, are emphasised [37]. Even with doses significantly higher than the standard currently recommended doses, reaching $1350 \mathrm{mg} /$ day [26] or even $2000 \mathrm{mg} /$ day [15], no adverse reactions or side effects were observed, and good tolerance of oral butyrate was underlined.

It can therefore be concluded that butyric acid is a substance with physiological action, showing a high safety level, both at the current standard doses and at significantly higher doses (four or six times higher). The need to increase the dose of butyrate supplementation is supported by many of the above-mentioned reasons, and such a dose increase is safe for the patient within the range specified above.

Short-chain fatty acids, including first of all butyric acid, are essential for the proper function of the gastrointestinal tract. This demand may increase significantly across the whole range of diseases and gastrointestinal dysfunctions, which justifies supplementation at higher doses than those used at present. The most important factors supporting such action are the following:

1. Physiological high demand of the epithelial cells for butyric acid, which is their primary energy substrate; current supplementation doses cover only a small proportion of the demand.

2. The trend observed in developed countries toward a decline in the production of endogenous SCFA, which may not even cover physiological needs.

3. A large number of pathological conditions or clinical situations that significantly increase the demand for SCFA, notably by enhancing the metabolism of the epithelial cells and their energy demand.

4. Frequent coexistence of these conditions and the greater demand of intestinal epithelial cells at concurrent reduction of the production of endogenous SCFA, including butyric acid. One can say about a sort of 'SCFA paradox' here: 'the more our digestive tract needs SCFA, the more difficult it is to provide substrates and to maintain normal microbiome to assure endogenous SCFA production'. 
5. Clinical and experimental observations confirming good effects of the use of high doses of butyrate in different pathological conditions.

6. Safety of high doses and no side effects or adverse reactions.

\section{Conflict of interest}

The authors whose names are listed immediately below certify that they have no affiliations with or involvement in any organization or entity with any financial interest, or non-financial interest in the subject matter or materials discussed in this manuscript.

\section{References}

1. Tan J, McKenzie C, Potamitis M, et al. The role of short-chain fatty acids in health and disease. Adv Immunol 2014; 121: 91-119.

2. Kuczyńska B, Wasilewska A, Biczysko M, et al. Krótkołańcuchowe kwasy tłuszczowe - mechanizmy działania, potencjalne zastosowania kliniczne oraz zalecenia dietetyczne [Short-chain fatty acids - mechanisms of action, potential clinical use and dietary recommendations]. Now Lek 2011; 80: 299-304.

3. Hague A, Butt AJ, Paraskeva C. The role of butyrate in human colonic epithelial cells: an energy source or inducer of differentiation and apoptosis? Proc Nutr Soc 1996; 55: 937-43.

4. Roediger WE. Role of anaerobic bacteria in the metabolic welfare of the colonic mucosa in man. Gut 1980; 21: 793-8.

5. Chambers ES, Preston T, Frost G, Morrison DJ. Role of gut microbiota-generated short-chain fatty acids in metabolic and cardiovascular health. Curr Nutr Rep 2018; 7: 198-206.

6. Sakata T. Pitfalls in short-chain fatty acid research: a methodological review. Anim Sci J 2019; 90: 3-13.

7. Kotunia A, Pietrzak P, Guilloteau P, et al. Butyric acid in the digestive tract. Gastroenterology Rev 2010; 5: 117-22.

8. Scheppach W, Christl SU, Bartram HP, et al. Effects of shortchain fatty acids on the inflamed colonic mucosa. Scand J Gastroenterol Suppl 1997; 222: 53-7.

9. Huang P, Liu Y. A reasonable diet promotes balance of intestinal microbiota: prevention of precolorectal cancer. Biomed Res Int 2019; 2019: 3405278.

10. Goverse G, Molenaar R, Macia L, et al. Diet-derived short chain fatty acids stimulate intestinal epithelial cells to induce mucosal tolerogenic dendritic cells. J Immunol 2017; 198: 2172-81.

11. Zhang WH, Jiang Y, Zhu QF, et al. Sodium butyrate maintains growth performance by regulating the immune response in broiler chickens. Br Poult Sci 2011; 52: 292-301.

12. Cleophas MCP, Ratter JM, Bekkering S, et al. Effects of oral butyrate supplementation on inflammatory potential of circulating peripheral blood mononuclear cells in healthy and obese males. Sci Rep 2019; 9: 775.

13. Hamer HM, Jonkers DM, Bast A, et al. Butyrate modulates oxidative stress in the colonic mucosa of healthy humans. Clin Nutr 2009; 28: 88-93.

14. Berni Canani R, Di Costanzo M, Leone L. The epigenetic effects of butyrate: potential therapeutic implications for clinical practice. Clin Epigen 2012; 4: 4.
15. Di Sabatino A, Morera R, Ciccocioppo R, et al. Oral butyrate for mildly to moderately active Crohn's disease. Aliment Pharmacol Ther 2005; 22: 789-94.

16. Pizzorno JE, Murray MT, Joiner-Bey H. Inflammatory bowel disease. In: The Clinician's Handbook of Natural Medicine. $3^{\text {rd }}$ edn. Churchill Livingstone 2016; 547-64.

17. Vernia P, Annese V, Bresci G, et al. Topical butyrate improves the efficacy of 5-ASA in refractory distal ulcerative colitis: results of a multicentre trial. Eur J Clin Invest 2003; 33: 244-8.

18. Hou Y, Wang L, Yi D, et al. Dietary supplementation with tributyrin alleviates intestinal injury in piglets challenged with intrarectal administration of acetic acid. Br J Nutr 2014; 111 : 1748-58.

19. Canani RB, Costanzo MD, Leone L, et al. Potential beneficial effects of butyrate in intestinal and extraintestinal diseases. World J Gastroenterol 2011; 17: 1519-28.

20. Haltmeier T, Inaba K, Schnüriger B, et al. Factors affecting the caloric and protein intake over time in critically ill trauma patients. J Surg Res 2018; 226: 64-71.

21. Yang $H$, Wang $X$, Xiong $X$, Yin Y. Energy metabolism in intestinal epithelial cells during maturation along the crypt-villus axis. Sci Rep 2016; 6: 31917.

22. Zhao XH, Yang T, Ma XD, et al. Heterogeneity of nutrition care procedures in nutrition guidelines for cancer patients. Clin Nutr 2019 Sep 9. pii: S0261-5614(19)33034-1. doi: 10.1016/j. clnu.2019.08.022

23. Nilaweera KN, Speakman JR. Regulation of intestinal growth in response to variations in energy supply and demand. Obes Rev 2018; 19 Suppl 1: 61-72.

24. Mitchell SE, Tang Z, Kerbois C, et al. The effects of graded levels of calorie restriction: I. impact of short term calorie and pro- tein restriction on body composition in the C57BL/ 6 mouse. Oncotarget 2015; 6: 15902-30.

25. Clausen MR, Bonnén H, Tvede M, Mortensen PB. Colonic fermentation to short-chain fatty acids is decreased in antibiotic-associated diarrhea. Gastroenterology 1991; 101: 1497-504.

26. Krokowicz L, Kaczmarek BF, Krokowicz P, et al. Sodium butyrate and short chain fatty acids in prevention of travellers' diarrhoea: a randomized prospective study. Travel Med Infect Dis 2014; 12: 183-8.

27. Chen Y, Liang J, Ouyang F, et al. Persistence of gut microbiota dysbiosis and chronic systemic inflammation after cerebral infarction in cynomolgus monkeys. Front Neurol 2019; 10: 661.

28. Haran JP, Bhattarai SK, Foley SE, et al. Alzheimer's disease microbiome is associated with dysregulation of the anti-inflammatory p-glycoprotein pathway. MBio 2019; 10: pii: e00632-19.

29. Hu Z, Guo Y. Effects of dietary sodium butyrate supplementation on the intestinal morphological structure, absorptive function and gut flora in chickens. Anim Feed Sci Technol 2007; 132: 240-9.

30. Yokoyama Y, Asahara T, Nomoto K, Nagino M. Effects of synbiotics to prevent postoperative infectious complications in highly invasive abdominal surgery. Ann Nutr Metab 2017; 71 Suppl 1: 23-30.

31. Couch RD, Dailey A, Zaidi F, et al. Alcohol induced alterations to the human fecal VOC metabolome. PLoS One 2015; 10: e0119362. 
32. Zeller I, Malovichko MV, Hurst HE, et al. Cigarette smoke reduces short chain fatty acid production by a Porphyromonas gingivalis clinical isolate. J Periodontal Res 2019; 54: 566-71.

33. Chow JY, Ma L, Zhu M, Cho CH. The potentiating actions of cigarette smoking on ethanol-induced gastric mucosal damage in rats. Gastroenterology 1997; 113: 1188-97.

34. Kieler IN, Osto M, Hugentobler L, et al. Diabetic cats have decreased gut microbial diversity and a lack of butyrate producing bacteria. Sci Rep 2019; 9: 4822..

35. Noureldein M, Bitar S, Youssef N, et al. Butyrate modulates diabetes-linked gut dysbiosis: epigenetic and mechanistic modifications. J Mol Endocrinol 2020; 64: 29-42.

36. Larasati RA, Harbuwono DS, Rahajeng E, et al. The role of butyrate on monocyte migration and inflammation response in patient with type 2 diabetes mellitus. Biomedicines 2019; 7. pii: E74.

37. Tarnowski W, Borycka-Kiciak K, Kiciak A, et al. Outcome of treatment with butyric acid In irritable bowel syndrome - preliminary report. Gastroenterol Prakt 2011; 1: 43-8.

Received: 14.05 .2020

Accepted: 15.05 .2020 